\title{
Complicações da via subcutânea na infusão de medicamentos e soluções em cuidados paliativos
}

\author{
Complications of the subcutaneous route in the infusion of medications and solutions in \\ palliative care
}

Natália de Almeida Barbosa Guedes ${ }^{1}$, Laís Samara de $\mathrm{Melo}^{1}$, Fernanda Batista Oliveira Santos ${ }^{1}$, Jaqueline Almeida Guimarães Barbosa ${ }^{1}$

Objetivo: caracterizar as complicações associadas ao uso da via subcutânea na infusão de medicamentos e soluções em cuidados paliativos. Métodos: estudo observacional, prospectivo, realizado na Unidade de Cuidados Paliativos de um hospital geral de ensino. Resultados: foram avaliados 78 pacientes em cuidados paliativos, nos quais foram realizadas 254 punções na via subcutânea para infusão de medicamentos e/ou soluções. A maioria era idosa $(87,3 \%)$ e encontrava-se desnutrida $(69,2 \%)$. Em 33,0\%, a via foi indicada para controle de sintomas e, em $50,0 \%$, a punção ocorreu na região anterolateral da coxa; $65,4 \%$ das punções não mostraram complicação. Dentre as complicações identificadas, 9,4\% foram edema e 9,1\% hiperemia, sendo que 53,8\% delas ocorreram na região deltoidea. A celulite ocorreu em apenas 3,5\%. Conclusão: a infusão de soluções pode ser considerada uma alternativa segura, a qual apresenta risco de complicações em sua maioria facilmente reversíveis e com baixo potencial de ocasionar danos aos pacientes.

Descritores: Cuidados de Enfermagem; Hipodermóclise; Cuidados Paliativos; Infusões Subcutâneas.

Objective: to characterize the complications associated with the use of the subcutaneous route for infusion of drugs and solutions in palliative care. Methods: observational, prospective study conducted at the Palliative Care Unit of a general teaching hospital. Results: the study analized seventy-eight patients in palliative care, in whom 254 punctures for infusion of drugs and/or solutions through the subcutaneous route were made. Most patients were elderly (87.3\%) and malnourished (69.2\%). In 33.0\%, the route was indicated for symptom control; in $50.0 \%$, the puncture occurred in the anterolateral thigh flap; $65.4 \%$ of the punctures showed no complication. Among the identified complications, 9.4\% were edema and 9.1\% hyperemia, and 53.8\% occurred in the deltoid region. Cellulite occurred in only $3.5 \%$. Conclusion: the infusion of solutions using the subcutaneous route can be considered a safe alternative, with risk of complications which are mostly reversible and with low potential to cause damage to patients.

Descriptors: Nursing Care; Hypodermoclysis; Palliative Care; Infusions, Subcutaneous.

$\overline{{ }^{1} \text { Universidade Federal de }}$ Minas Gerais. Belo Horizonte, MG, Brasil. 


\section{Introdução}

O envelhecimento populacional e o aumento da prevalência do câncer e de outras doenças não transmissíveis como a hipertensão, o diabetes mellitus e as demências têm gerado a necessidade de reformulação do cuidado em saúde e também do aprimoramento de técnicas que possam beneficiar esses pacientes, considerando as especificidades dessa população ${ }^{(1)}$. Uma vez hospitalizados, os esforços para o cuidado dos pacientes voltam-se quase sempre para intervenções em busca de cura, o que requer, muitas vezes, a utilização de métodos invasivos, que nem sempre resultam em benefícios. São abordagens que constantemente se mostram desproporcionais às necessidades e possibilidades dos pacientes e que pouco consideram o sofrimento que ocasionam ${ }^{(2)}$.

Diante desse contexto, o oferecimento de cuidados paliativos tem ganhado cada vez mais respaldo, focando a terapêutica nas necessidades apresentadas de forma proporcional à evolução natural da doença. O conceito de cuidados paliativos foi definido pela Organização Mundial da Saúde em 1990 e este era direcionado apenas aos pacientes com câncer visando aos cuidados com fim da vida. No ano de 2002, este foi revisto e ampliado, passando a considerar também essa assistência a pacientes com outras doenças crônicas e degenerativas. Em 2004, um novo documento foi divulgado pela Organização Mundial de Saúde, considerando os cuidados paliativos como aqueles capazes de melhorar a qualidade de vida de pacientes e seus familiares que enfrentam doenças que ameacem a vida por meio da prevenção e alívio do sofrimento. Essa abordagem amplia o horizonte de ação dos cuidados paliativos, não restringindo-se a protocolos, e sim à individualidade dos contextos desses pacientes e seus familiares ${ }^{(3)}$.

Na modalidade de cuidados paliativos, a via oral será a de escolha para a administração de medicamentos em razão de ser a mais simples e a de menor invasividade. Nos casos em que a via oral não se encontra viável, uma vez que seu uso poderia ocasionar danos aos pacientes, outras vias serão consideradas. Até $70,0 \%$ dos pacientes que enfrentam quadros de doenças ameaçadoras da vida precisarão de uma via alternativa para administração de medicamentos, em geral por redução do nível de consciência e incapacidade de deglutição( ${ }^{(4)}$.

Assim, a via subcutânea apresenta-se como uma opção para administração de medicamentos e fluidos quando a via oral está contraindicada, como nos pacientes que sofrem com disfagia, náuseas e vômitos persistentes, constipação intestinal e diarreia, permitindo a correção de quadros de desidratação que não exija pronta reposição de volume ${ }^{(5)}$. Pacientes com confusão mental e dispneia grave também podem ser beneficiados pelo uso da via subcutânea ${ }^{(4)}$.

A via subcutânea é considerada mais confortável e menos dolorosa para o paciente, quando comparada à punção venosa, e, além disso, por ser de fácil inserção também diminui a ocorrência de inúmeras tentativas sem sucesso para punção. Embora o manejo assistencial da via subcutânea seja considerado uma prática segura, esta não é isenta de riscos. Em recente estudo de revisão integrativa, encontrou-se que dentre os principais efeitos adversos relacionados ao uso de hipodermóclise (infusão de soluções no tecido subcutâneo) estão a dor e edema, celulite e absorção insuficiente da solução com acúmulo de líquido no local da punção ${ }^{(6)}$. São riscos considerados simples por não trazerem grandes prejuízos aos pacientes, mas que só serão minimizados ou evitados se forem conhecidos e problematizados nas esferas da assistência, do ensino e da pesquisa, considerando que em cuidados paliativos o arcabouço norteador do processo cuidativo centra-se no bem-estar e no alívio do sofrimento dos pacientes.

A despeito de suas vantagens, como pela maior facilidade de punção e menor risco de complicações graves $^{(7)}$, o uso da via subcutânea para infusão de medicamentos e soluções ainda é considerado uma prática subutilizada na clínica assistencial. Assim, a realização de estudos em relação ao procedimento se faz necessária, motivando o desenvolvimento des- 
ta pesquisa. Sendo assim, as questões que nortearam a realização desta investigação foram: Quais são as principais indicações, sítios de punção e tempo de durabilidade do acesso na via subcutânea? Existem fatores que podem influenciar a ocorrência de complicações ao se utilizar essa via?

Neste contexto, este estudo teve como objetivo caracterizar as complicações associadas ao uso da via subcutânea na infusão de medicamentos e soluções em cuidados paliativos. A pesquisa corrobora com o avanço no conhecimento sobre o uso da via subcutânea em pacientes que estão em cuidados paliativos e, com isso, para a ampliação dessa prática de forma segura e efetiva para pacientes, profissionais e serviços de saúde.

\section{Métodos}

Estudo observacional, prospectivo. Foi realizado acompanhamento de todos os pacientes internados em uso da via subcutânea para infusão de medicamentos e soluções em uma unidade de cuidados paliativos ao longo de quatro meses (maio a agosto de 2018). Os critérios de inclusão foram pacientes com idade superior a 18 anos, internados na unidade de cuidados paliativos, em uso da via subcutânea para infusão de medicamentos e soluções. Foram excluídos procedimentos que haviam sido feitos previamente à internação na unidade de cuidados paliativos.

O estudo foi realizado em um hospital geral, público, de grande porte e de ensino. A instituição conta com uma unidade exclusiva destinada a cuidados paliativos, com 16 leitos. Foram atendidos em 2018 uma média de 50 pacientes por mês, oriundos de Belo Horizonte e região metropolitana. Os dados foram coletados pela pesquisadora residente de enfermagem, que estava presente no serviço de segunda a sexta-feira. Nos fins de semana, o acompanhamento dos pacientes era realizado pelos enfermeiros de plantão, os quais foram orientados a fazer os registros necessários. Vale ressaltar que todos os enfermeiros que trabalham na unidade possuem treinamento sobre os cuidados e uso da via subcutânea, fornecido pela instituição, sendo considerados aptos a identificar a presença de complicações.

Foram coletados dados sociodemográficos e clínicos dos prontuários como sexo, idade, estado nutricional, diagnóstico de mau prognóstico e desfecho dos pacientes acompanhados a fim de caracterizar a população do estudo. Na modalidade de cuidados paliativos, adotou-se o diagnóstico definidor de mau prognóstico considerando a possibilidade de indicação para aqueles pacientes com quadros de terminalidade, com dor e/ou sofrimento importantes ${ }^{(8-9)}$.

Pacientes em uso de hipodermóclise e que atendiam aos critérios de elegibilidade foram diariamente avaliados, uma vez ao dia, sendo que as informações relativas à punção eram registradas em um instrumento de coleta de dados elaborado pelas autoras, adaptado do instrumento formulário utilizado pelo Hospice do Hospital das Clínicas de São Paulo - Jaçanã para avaliação de hipodermóclises. Os pacientes foram acompanhados até receberem alta, apresentarem óbito ou deixarem de usar a via subcutânea como via para administração de medicamentos e soluções.

Foram coletados dados relativos ao procedimento durante a observação diária, tais como indicação do uso da via; local da punção; medicamentos infundidos na via; duração da punção; dispositivo utilizado na punção; tipo de fixação utilizada. Além disso, foi levantada a ocorrência de complicações, as quais foram classificadas por tipo de complicação (hiperemia, edema, celulite, sangramento, dor, abcesso, necrose) e o sítio de ocorrência. Alguns dados foram completados por meio de consulta ao prontuário eletrônico, ao próprio paciente ou familiar presente.

Posteriormente, foram realizadas análises estatísticas de distribuição de frequência e medidas descritivas utilizando o Statistical Package for the Social Sciences, versão 23. Foram feitas também análises estratificadas buscando possíveis correlações entre as variáveis. Nas análises descritivas dos pacientes, cada um deles foi contado apenas uma vez $(n=78)$. Quanto às punções, cada uma foi analisada de maneira inde- 
pendente, sendo possível, assim, um mesmo paciente gerar mais de um dado de punção $(n=254)$. Nas análises de punções, não foram consideradas dependências/influências entre punções em um mesmo paciente. Nas análises de correlações, foi verificado se a relação entre duas ou mais variáveis tinha significância estatística, sendo que foi considerada uma significância de 95\%. Situações em que os valores obtidos não atingiram os $95 \%$ não foram apresentadas.

O estudo foi aprovado pelo Comitê de Ética e Pesquisa da Universidade Federal de Minas Gerais com o parecer no $2.745 .989 / 2018$.

\section{Resultados}

Foi realizado acompanhamento de 78 pacientes, os quais foram submetidos a 254 punções na via subcutânea para infusão de medicamentos e soluções durante o período do estudo. Destes, 42 (53,8\%) eram mulheres e $36(46,2 \%)$ eram homens. A idade média foi de 78 anos com desvio padrão de 13,5. A maioria dos pacientes, 38 (48,7\%), tinha idade entre 60 e 79 anos; 30 (38,6\%) tinham idade maior que 80 anos; e $10(12,8 \%)$ pacientes tinham idade entre 40 e 59 anos. 0 diagnóstico de estado nutricional foi retirado do prontuário de registros médicos em que 54 (69,2\%) foram considerados desnutridos, 14 (17,9\%) eutróficos, $6(7,7 \%)$ obesos e $4(5,1 \%)$ não havia registro de estado nutricional no prontuário.

Na população pesquisada, prevaleceram como definidores de mau prognóstico a demência avançada, 25 (32\%), seguida por doença oncológica, 17 (21,7\%), fragilidade, 17 (21,7\%), doença neurológica, 12 (15,3\%), falência orgânica, 4 (5,1\%), e outros, 3 $(3,8 \%)$. A maior parte dos participantes do estudo, 73 $(93,6 \%)$, encontrava-se em fase final de vida. Quanto ao desfecho dos pacientes atendidos, $41(52,6 \%)$ foram a óbito, $31(41,0 \%)$ receberam alta hospitalar e 5 $(6,4 \%)$ foram transferidos para outra instituição.

No que se refere à indicação para o uso da via subcutânea, esta foi usada para controle de sintomas em 84 situações $(33,0 \%)$ devido ao difícil acesso à rede venosa periférica em 66 (25,9\%), como via de hidratação em 47 (18,5\%) e em 29 vezes (11,4\%) ocorreu por outros motivos, como em situações em que a medicação infundida exigia via exclusiva.

Dentre as 254 instalações realizadas na via subcutânea, em 127 (50,0\%) a punção foi realizada na região anterolateral da coxa, em $61(24,0 \%)$ na região abdominal, em 52 (20,5\%) na região do deltoide, em 11 (4,3\%) na região infraclavicular e em $3(1,2 \%)$ na região interescapular. Em todas as situações, o dispositivo de escolha foi o cateter não agulhado, de número 22 ou 24. Em 250 (98,4\%) punções, a fixação foi realizada com filme transparente estéril e em 4 (1,5\%) punções foi utilizada fita adesiva microporosa. A duração de cada punção variou entre inferior às $24 \mathrm{~h}$ e seis dias, com média de 3,5 dias de permanência.

0 principal motivo para a retirada ou substituição da punção na via subcutânea, em 89 (35\%), foi o fato de ter chegado ao período normatizado para troca na Instituição - cinco dias. Trocas por motivos de complicações estiveram presentes em 41 (16,1\%) situações acompanhadas. Outras causas para a retirada da punção na via subcutânea foram: óbito do paciente, 44 (17,3\%); dispositivo solto durante a manipulação, 21 (8,3\%); dispositivo retirado pelo paciente, 21 (8,3\%); alta hospitalar do paciente, 18 (7,1\%); dispositivo retirado conforme prescrição médica, 13 (5,1\%); e sem registro do motivo da retirada, 7 (2,8\%).

Dentre as soluções e medicações administradas na via subcutânea, soroterapia (cloreto de sódio 0,9\%), com aporte calórico ou em combinação com outras medicações, foi administrada em 155 (61,0\%) situações. 0 fármaco mais infundido foi a morfina, prescrito em 124 procedimentos (48,8\%), seguido por dipirona em 82 procedimentos $(32,3 \%)$ e a escopolamina que foi administrada em 58 procedimentos $(22,8 \%)$. As demais medicações infundidas e que apareceram em menos que $20 \%$ dos procedimentos foram: haldol (16,5\%); metoclopramida $(15,7 \%)$; ondansetrona $(9,8 \%)$; ceftriaxona e meropenem $(9,4 \%$ cada); omeprazol $(8,7 \%)$; furosemida $(8,3 \%)$; dexametasona $(7,1 \%)$; ranitidina $(5,9 \%)$; midazolam 
(5,1\%); teicoplanina $(4,3 \%)$; fenobarbital $(3,9 \%)$; cefepime $(3,1 \%)$; tramadol $(1,6 \%)$; metadona $(1,2 \%)$; e ampicilina $(0,8 \%)$.

Dentre as 254 punções realizadas, na maioria das vezes $(65,4 \%)$ não houve nenhuma complicação. Dos $34,6 \%$ que tiveram complicações, prevaleceram o edema $(9,4 \%)$ e a hiperemia $(9,1 \%)$ entre as complicações. Ao analisar a ocorrência das complicações relacionadas ao local de punção, encontrou-se que, proporcionalmente, as punções realizadas na região do deltoide foram as que mais complicaram. Das 52 punções realizadas, 28 (53,8\%) apresentaram algum tipo de complicação.

A ocorrência de complicações nas demais regiões foram: região anterolateral da coxa, 127 punções realizadas e 40 complicações identificadas (31,5\%); abdome, 61 punções realizadas e 19 complicações identificadas (31,1\%); região subclavicular, 11 punções realizadas e uma complicação identificada (9,1\%); na região interescapular foram realizadas 3 punções e nenhuma complicação identificada.

Na Tabela 1 estão descritas as complicações observadas em cada local de punção, tendo prevalecido o edema na região do deltoide $(35,7 \%)$, seguido por hiperemia na região da coxa $(32,5 \%)$. A ocorrência de celulite foi maior na região da coxa $(12,5 \%)$.

Tabela 1 - Tipo de complicação por local de punção

\begin{tabular}{|c|c|c|c|c|c|c|c|}
\hline Local & $\begin{array}{c}\text { Edema } \\
(\%)\end{array}$ & $\begin{array}{l}\text { Hipere- } \\
\text { mia(\%) }\end{array}$ & $\begin{array}{l}\text { Dor } \\
\text { (\%) }\end{array}$ & $\begin{array}{c}\text { Celulite } \\
\text { (\%) }\end{array}$ & $\begin{array}{c}\text { Sangra- } \\
\text { mento } \\
(\%)\end{array}$ & $\begin{array}{c}\text { Hema- } \\
\text { toma } \\
(\%)\end{array}$ & $\begin{array}{c}\text { Outros } \\
\text { (\%) }\end{array}$ \\
\hline Deltoide & 35,7 & 14,2 & 17,8 & 7,1 & - & - & 25,0 \\
\hline Coxa & 22,5 & 32,5 & 10,0 & 12,5 & 2,5 & 2,5 & 17,5 \\
\hline Abdome & 26,3 & 31,5 & 15,7 & 10,5 & - & - & 15,7 \\
\hline
\end{tabular}

A Tabela 2 mostra as principais medicações infundidas em punções identificadas com complicações, nas quais uma maior prevalência de complicações associadas ao uso de ranitidina e metoclopramida pode ser observada.
Tabela 2 - Complicações relacionadas aos medicamentos infundidos na via subcutânea

\begin{tabular}{lccccccc}
\hline Medicamento & $\begin{array}{c}\text { Hipere- } \\
\text { mia(\%) }\end{array}$ & $\begin{array}{c}\text { Sangra- } \\
\text { mento(\%) }\end{array}$ & $\begin{array}{c}\text { Celuli- } \\
\text { te(\%) }\end{array}$ & $\begin{array}{c}\text { Dor } \\
\text { (\%) }\end{array}$ & $\begin{array}{c}\text { Ede- } \\
\text { ma(\%) ções(\%) }\end{array}$ & $\begin{array}{c}\text { Pun- } \\
\text { çóes(\%) }\end{array}$ \\
\hline Ranitidina & 40,0 & 6,6 & 20,0 & & & 15 & 53,3 \\
Cefepime & - & - & - & 25,0 & 37,5 & 8 & 37,5 \\
Teicoplanina & 18,1 & - & - & - & - & 11 & 36,3 \\
$\begin{array}{l}\text { Metoclopra- } \\
\text { mida }\end{array}$ & 22,5 & - & 15,0 & 15,0 & 17,5 & 40 & 30,0 \\
Ceftriaxona & 12,5 & - & - & 4,1 & 16,6 & 24 & 25,0 \\
Meropenem & 12,5 & - & - & 4,1 & 4,1 & 24 & 25,0 \\
\begin{tabular}{l} 
Dipirona \\
\hline
\end{tabular} & 14,6 & 1,2 & 6,1 & 8,5 & 14,6 & 82 & 24,3 \\
\hline
\end{tabular}

\section{Discussão}

O estudo apresenta algumas limitações como a impossibilidade de se calcular medidas preditivas que favoreçam melhores práticas de cuidados, bem como de se determinar a causalidade das complicações advindas do uso da via subcutânea na infusão de medicamentos e soluções em pacientes em cuidados paliativos. Contudo, propicia achados que contribuem para a elucidação do uso dessa modalidade terapêutica.

Os participantes do estudo caracterizaram-se por apresentar um perfil social e clínico preocupante por ser favorecedor da ocorrência de complicações do seu estado de saúde como um todo, sendo a maioria de idosos desnutridos. Dentre eles, $21,7 \%$ encontram-se com neoplasia, perfil esse observado em outros estudos realizados com pacientes em cuidados paliativos. A indicação dessa modalidade terapêutica para pacientes oncológicos é consistente e mundialmente conhecida devido ao fato do câncer repercutir em aspectos biopsicossociais na vida da pessoa e de sua família, em especial quando a doença se apresenta em estágio avançado e com metástases, com baixa possibilidade terapêutica de cura(10-12).

No entanto, em pacientes com outros diagnósticos, os cuidados paliativos também se fazem necessários com o objetivo de promover a condição de bem-estar global, como os agravos neurológicos, presentes 
na maioria dos participantes do estudo. Nos casos de demência avançada, as intervenções previstas nos cuidados paliativos também se mostram como importantes na medida em que são identificadas as evidências de alterações estruturais e comprometimentos funcionais $^{(8,13)}$.

A fragilidade mostrou-se o principal definidor de mau prognóstico. A literatura pontua que idosos em condições de fragilidade intensa, mesmo que sem diagnóstico específico, mas que demonstram estar em declínio progressivo de vitalidade e aparentam desenvolver uma trajetória previsível em direção à morte dentro dos próximos meses, também têm indicação de cuidados paliativos a fim de melhorar sua qualidade de vida ${ }^{(8,14)}$.

0 percentual de óbitos foi menor que o encontrado em outros serviços de cuidados paliativos, geralmente superior a $70,0 \%{ }^{(10)}$. Acredita-se que o percentual de pacientes com desfecho de alta hospitalar, apesar do quadro clínico em que predominaram agravos complexos e fragilidade, decorra de efetivo controle de sintomas, sinalizando qualidade assistencial e a efetividade dos cuidados paliativos para essa população.

Em relação à indicação da via subcutânea, os resultados obtidos corroboram com estudo realizado com 16 pacientes em cuidados paliativos, no qual as indicações para a via foram o controle de sintomas como a disfagia, náuseas e vômitos, agitação e prostração ${ }^{(15)}$. Além disso, tal via tem se mostrado muito utilizada para hidratação, tendo em vista o alto risco de desidratação na população com esse perfil clínico ${ }^{(4)}$.

Sobre a perspectiva da hidratação, um estudo inglês ${ }^{(16)}$ apontou que a via subcutânea fornece uma série de vantagens sobre a intravenosa para indivíduos incapazes de tolerar fluidos entéricos e com necessidade de hidratação, pois está associada a menos complicações, bem como uma maior variedade de locais de infusão. Isso a torna particularmente vantajosa para pacientes frágeis e/ou idosos que necessitam de hidratação de baixo volume, podendo ser administra- dos fluidos com segurança, inclusive no ambiente domiciliar.

No que diz respeito aos locais de punção, a literatura indica como locais mais adequados a região subclavicular, região interescapular, região abdominal (flancos), anterolateral da coxa e região deltóidea como as mais indicadas ${ }^{(4)}$, corroborando com os achados deste estudo. 0 alto percentual de punções na região anterolateral da coxa decorre do estado nutricional deficitário da maioria dos pacientes assistidos.

Trata-se de regiões com maior camada de tecido subcutâneo, além de serem regiões com maior capacidade para receber grandes volumes, salientando ser a hidratação uma importante indicação do uso da via. Ressalta-se que na escolha do local da punção é preciso levar em consideração o volume de líquido a ser administrado, sendo as regiões abdominal e anterolateral da coxa boas opções nessas situações ${ }^{(4)}$, pontuando que a hidratação foi um dos fatores predominantes para o uso da via.

A escolha pelo uso do cateter não agulhado se dá por ser flexível e apresentar menor risco de trauma cutâneo para o paciente, bem como menor risco de acidente de trabalho para o profissional ${ }^{(15)}$. Quanto à fixação do mesmo, o filme transparente estéril é preconizado na instituição e está de acordo com as recomendações mais recentes ${ }^{(4)}$, o qual, além de proteger e evitar a ocorrência de infecções no local da punção, permite a visualização do sítio de punção a todo momento. Além disso, contribui para a prevenção da perda do dispositivo.

Quanto ao tempo médio de permanência do dispositivo, o protocolo institucional recomenda que a troca de dispositivo seja feita quando este completar 5 dias. A extensão do prazo recomendado pode ocorrer em situações atípicas, avaliadas pela equipe de enfermagem. Na literatura não há consenso acerca do tempo máximo de permanência da mesma punção. A recomendação é de que, havendo indícios de alguma complicação, ainda que a punção seja recente, a mesma seja retirada e uma nova punção seja realizada, a 
uma distância mínima de cinco centímetros do local anterior ${ }^{(4)}$. Retiradas de punção em decorrência de complicações correspondeu à minoria das situações, dando indícios da segurança no uso desse recurso no tratamento em saúde.

Quanto às complicações no uso da via subcutânea para infusão de medicamentos e soluções, prevaleceram o edema e hiperemia, as quais se caracterizam por serem de baixa gravidade, reversíveis e com pouca repercussão clinica para o paciente, necessitando apenas da redução da velocidade de infusão ou realizando troca do local de punção ${ }^{(6)}$, conduta essa adotada na Instituição. A celulite foi a complicação de maior gravidade, tendo ocorrido, contudo, em número muito pequeno de situações $(3,5 \%)$. Ela requer o uso de antibióticos, compressas frias e acompanhamento diário do local afetado ${ }^{(4)}$.

As punções realizadas na região do deltooide foram as que mais complicaram, manifestando-se por meio de edema. Acredita-se decorrer pela menor quantidade de tecido subcutâneo quando comparado com as regiões do abdome e anterolateral da coxa. Esse local deve ser evitado para infusões contínuas de solução como soroterapia ou ser utilizado apenas quando não for possível a punção em outros locais com maior quantidade de tecido subcutâneo ${ }^{(4)}$.

A soroterapia (cloreto de sódio 0,9\%), com ou sem aporte calórico ou em combinação com outras medicações, e a morfina predominaram entre os líquidos administrados, o que corrobora com achados de outros estudos $^{(15,17)}$. As demais medicações infundidas também estão descritas em outros trabalhos realizados com pessoas em cuidados paliativos. Um deles apontou que os medicamentos mais utilizados por essa via foram morfina, escopolamina, metoclopramida, cefepime, ondansetrona, cloreto de sódio 0,9\%, dipirona, haloperidol, clorpromazina, dexametasona e furosemida ${ }^{(17)}$.

Contudo, os achados não permitem conclusões sobre a ocorrência de complicações em relação aos medicamentos em uso pelos pacientes, sendo necessários novos estudos. Cabe pontuar que as soluções melhor toleradas são as isotônicas, as hidrossolúveis e aquelas com $\mathrm{pH}$ próximo à neutralidade por causarem menos reações irritantes ${ }^{(4,16-18)}$.

A realização do uso da via subcutânea para a infusão de medicamentos e soluções mostra-se, diante dos resultados encontrados, uma alternativa viável e efetiva, passível de ser usada inclusive em outras situações que não exclusivamente para tratamento paliativo, sendo o procedimento de responsabilidade da equipe de enfermagem. Cabe a toda a equipe multiprofissional analisar as diferentes situações em que essa via pode ser utilizada, bem como os resultados obtidos, considerando suas complicações e suas relações, a fim de evitá-las, oferecendo um cuidado que considere as condições singulares do paciente, em uma abordagem individualizada e integral ${ }^{(19-20)}$.

\section{Conclusão}

Os resultados encontrados neste estudo sinalizam que o uso da via subcutânea para infusão de medicamentos e soluções pode ser considerada uma alternativa de infusão segura, a qual apresenta risco de complicações em sua maioria facilmente reversíveis e com baixo potencial de ocasionar danos aos pacientes. A equipe de enfermagem precisa ser continuadamente capacitada para a redução da ocorrência de complicações, mesmo que leves. Sugere-se a realização de novos estudos que contribuam com análises mais aprofundadas relacionadas à causalidade das complicações no uso da via subcutânea.

\section{Agradecimentos}

Agradecemos ao Hospital Risoleta Tolentino Neves por meio da Residência Multiprofissional.

\section{Colaborações}

Guedes NAB colaborou com a concepção, projeto, análise e interpretação dos dados, redação do artigo e revisão crítica relevante do conteúdo intelec- 
tual. Melo LS, Santos FBO e Barbosa JAG colaboraram com a redação do artigo, revisão crítica relevante do conteúdo intelectual e aprovação final da versão a ser publicada.

\section{Referências}

1. Saad PM. Envelhecimento populacional: demandas e possibilidades na área de saúde. Series Demográficas. ABEP [Internet]. 2016 [citado 2019 ago. 10]; 3:153-66. Disponível em: http://www. abep.org.br/ abeporgb/publicacoes/index.php/ series/article/viewFile/71/68

2. Sociedade Brasileira de Geriatria e Gerontologia. Vamos falar de cuidados paliativos [Internet]. 2016 [citado 2019 fev 18]. Disponível em: http:// sbgg.org.br/wp-content/uploads/2014/11/ vamos-falar-de-cuidados-paliativos-vers--oonline.pdf

3. World Health Organization. WHO definition of palliative care [Internet]. 2010 [cited Feb 22, 2019]. Available from: http://www.who.int/ cancer/palliative/ definition/en accessed

4. Azevedo DL. O uso da via subcutânea em geriatria e cuidados paliativos: um guia da SBGG e da ANCP para profissionais. Rio de Janeiro: SBGG; 2017.

5. Riegel F, Costa KC, Oliveira Junior J, Migowski ER, Siqueira DS. Eficácia da hipodermóclise na administração de medicamentos em cuidados paliativos. Rev Enferm UFPI [Internet]. 2018 [citado 2019 jan. 08]; 7(2):64-71. Disponível em:http://www.ojs.ufpi.br/index.php/reufpi/ article/view/6807

6. Nunes PMSA, Souza RCS. Adverse effects of hypodermoclysis in adult patients: an integrative review. Rev Min Enferm. 2016; 20:e951. doi: http://dx.doi.org/10.5935/1415-2762.20160020

7. Gomes NS, Silva AMB, Zago LB, Lima e Silva EC, Barichello E. Nursing knowledge and practices regarding subcutaneous fluid administration. Rev Bras Enferm. 2017; 70(5):1096-105. doi: http:// dx.doi.org/10.1590/0034-7167-2016-0424

8. Rodrigues LA, Ligeiro C, Silva MD. Cuidados paliativos, diagnósticos e terminalidade: indicação e início do processo de paliação [Internet]. 2015 [citado 2019 jan. 08]; 9(1):26-35. Disponível em: http://fundacaopadrealbino.org.br/facfipa/ner/ pdf/Revistacuidarteenfermagem $\% 20 v . \% 209 \% 20$ n.1\%20\%20jan.\%20jun\%202015.pdf

9. Bezerra S, Mauriz P. Protocolo de cuidados paliativos multiprofissional do Hospital Regional do Cariri [Internet]. 2015 [citado 2019 abr 06]. Disponível em: http://paliativo.org.br/revista/ revista-ancp-ed2.pdf

10. Oliveira HAD, Freitas IMD, Braga PG, Santos POO, Alcântara CO, Cintra MTG, et al. Análise do uso de terapia invasivas em pacientes oncológicos em cuidados paliativos internados em dois hospitais públicos gerais de Belo Horizonte. Rev Med Minas Gerais. 2018; 28(Supl 5):280515. doi: http:// www.dx.doi.org/10.5935/2238-3182.20180127

11. Figueiredo JF, Souza VM, Coelho HV, Souza RS. Qualidade de vida de pacientes oncológicos em cuidados paliativos. Rev Enferm Centro-Oeste Min. 2018; 8:e2638. doi: http://dx.doi.org/10.19175/ recom.v8i0.2638

12. Silva RS, Oliveira CC, Pereira Á, Amaral JB. The treatment of terminally ill patients in the perception of nursing undergraduates. Rev Rene. 2015; 16(3):415-24. doi: http://dx.doi. org/10.15253/2175-6783.2015000300015

13. Abreu WC. Cuidados paliativos para utentes com demência avançada: reflexões sobre a sua implementação. Rev Port Enferm Saúde Mental. 2016; (16):6-10. doi: http://dx.doi.org/10.19131/ rpesm.0151

14. Bakitas MA, Tosteson TD, Li Z, Lyons KD, Hull JG, Li Z, et al. Early versus delayed initiation of concurrent palliative oncology care: patient outcomes in the ENABLE III randomized controlled trial. J Clin Oncol. 2015; 33(13):1438-45. doi: http://dx.doi. org/10.1200/JC0.2014.58.6362

15. Adriani PA, Siqueira AC, Barbosa AF, Carmo JS, Pontes NS, Rocha VPS. A aplicação da hipodermóclise em pacientes durante os cuidados paliativos. Uniĺtalo Pesqu [Internet]. 2016 [citado 2019 mar 22]; 6(2):65-89. Disponível em: http:// pesquisa.italo.br/index.php?journal=uniitalo\&pa ge=article \&op=view\&path $\% 5 B \% 5 \mathrm{D}=57 \&$ path $\%$ $5 \mathrm{~B} \% 5 \mathrm{D}=57$

16. Gabriel J. Subcutaneos fluid administration and the hydration of older people. Br J Nurs. 2014 [cited July 24, 2019];23(suppl 14):10-4. 
Available from: http://www.ncbi.nlm.nih.gov/ pubmed/25062366

17. Beales A. An innovative approach to hydration for a patient with dementia. Nurs Older People. 2017; 29(4):26-29. http://dx.doi.org/10.7748/ nop.2017.e898

18. Quaglio RC, Varallo FR, Lima NKC, Junqueira AF, Júnior EI, Matumoto S, Fortuna CM. Medicamentos passíveis de infusão por hipodermóclise. Medicina (Ribeirão Preto). 2018; 51(1):55-68. doi: http://dx.doi.org/10.11606/issn.2176-7262. v51i1p55-68
19. Lima MPO, Oliveira MCX. Meanings of nursing care for relatives of patients receiving palliative care. Rev Rene. 2015; 16(4):593-602. doi: http://dx.doi. org/10.15253/2175-6783.2015000400017

20. Kavalieratos D, Corbelli J, Zhang D, Dionne-Odom JN, Ernecoff NC, Hanmer J, et al. Association between palliative care and patient and caregiver outcomes: a systematic review and meta-analysis. JAMA. 2016; 316(20):2104-14. doi: http:// dx.doi.org/10.1001/jama.2016.16840 